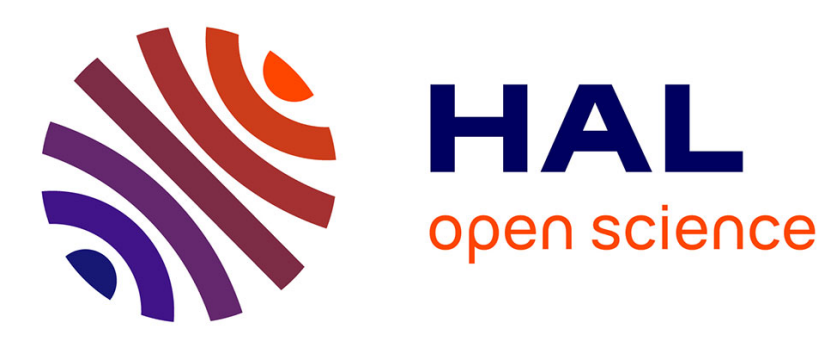

\title{
Dispositif d'étude des surfaces solides ou liquides par réflexion spéculaire des rayons $\mathrm{X}$ \\ Louis Bosio, Robert Cortès, Guy Folcher, M. Oumezine
}

\section{To cite this version:}

Louis Bosio, Robert Cortès, Guy Folcher, M. Oumezine. Dispositif d'étude des surfaces solides ou liquides par réflexion spéculaire des rayons X. Revue de Physique Appliquée, 1985, 20 (6), pp.437-443. 10.1051/rphysap:01985002006043700 . jpa-00245356

\section{HAL Id: jpa-00245356 https://hal.science/jpa-00245356}

Submitted on 1 Jan 1985

HAL is a multi-disciplinary open access archive for the deposit and dissemination of scientific research documents, whether they are published or not. The documents may come from teaching and research institutions in France or abroad, or from public or private research centers.
L'archive ouverte pluridisciplinaire HAL, est destinée au dépôt et à la diffusion de documents scientifiques de niveau recherche, publiés ou non, émanant des établissements d'enseignement et de recherche français ou étrangers, des laboratoires publics ou privés. 


\title{
Dispositif d'étude des surfaces solides ou liquides par réflexion spéculaire des rayons $\mathbf{X}$
}

\author{
L. Bosio, R. Cortès, G. Folcher et M. Oumezine \\ Laboratoire CNRS "Physique des Liquides et Electrochimie », associé à l'Université Pierre et Marie Curie, \\ T. 22, 4, place Jussieu, 75230 Paris Cedex 05, France
}

(Reçu le 19 septembre 1984, révisé le ler février 1985, accepté le 25 février 1985)

\begin{abstract}
Résumé. - On décrit un dispositif permettant de tester des modèles de profils de densité au voisinage d'une interface solide (ou liquide) -vapeur. Le principe de la méthode consiste à mesurer, en fonction de l'angle d'attaque (de l'ordre de quelques mrad), l'intensité réfléchie spéculairement par une surface plane lorsque celle-ci reçoit un faisceau de rayons $\mathrm{X}$. On donne quelques exemples d'application.
\end{abstract}

\begin{abstract}
An apparatus for X-ray reflection measurements is described. The goniometer is designed for studies on liquid and solid surfaces at grazing angles in the range 0-100 mrad. Typical examples using this set up are reported.
\end{abstract}

\section{Introduction.}

On dispose à présent de méthodes d'analyse des surfaces extrêmement performantes et en nombre tel qu'il serait fastidieux d'en dresser une liste. Parmi celles-ci, les techniques de diffraction et de spectroscopie électroniques sont certainement les plus utilisées lorsque l'on désire obtenir des informations à l'échelle atomique mais la nécessité d'opérer sous vide limite parfois leur champ d'application. Les méthodes optiques sont souvent mises en ouvre pour étudier in situ les interfaces; cependant, les études effectuées à l'interface mercure liquide-vapeur ont conduit à une description contradictoire de la zone de transition [1].

Puisque les méthodes de diffraction conventionnelles des rayons $X$ ou des neutrons ne peuvent convenir en raison de la trop grande profondeur de pénétration des rayonnements dans la matière, on assiste au développement de techniques qui tentent de pallier cet inconvénient en utilisant l'incidence rasante $[2,3]$ ou une réflexion de Bragg [4] : la profondeur de pénétration peut alors être réduite à quelques nanomètres. Dans certains cas favorables ces méthodes fournissent des informations sur l'ordre local, sur la structure à plus longue portée ou sur l'insertion d'atomes à la surface d'une matrice.

Il est également possible de tester des modèles de profils de densité dans la zone superficielle (c'est-à-dire la variation de la densité en fonction d'une direction perpendiculaire à la surface) à condition de connaître la courbe de réflectivité spéculaire jusqu'à des angles d'attaque plusieurs fois plus élevés que l'angle critique $\theta_{c}$ de réflexion "totale ". C'est cette méthode que nous utiliserons ici et, bien qu'elle puisse être envisagée pour divers types de rayonnements [5], nous ne considérerons que la réflexion des rayons $\mathrm{X}$.

Connue depuis Compton [6], la réflectivité des rayons $\mathrm{X}$ sur des surfaces solides a été appliquée à la mesure des épaisseurs de films déposés sur des substrats [7] puis à l'étude des rugosités ou des inhomogénéités de surface $[8,9]$. Si l'on fait abstraction des mesures de réflexion sur les surfaces liquides effectuées par Steps [10] - et dans lesquelles le but n'était pas d'étudier l'interface liquide-vapeur c'est à Rice et à ses collaborateurs que revient le mérite d'avoir proposé [11] puis réalisé $[11,12]$ les premières études de réflectivité des rayons $\mathbf{X}$ pour déterminer le profil de densité à la surface des métaux liquides. On peut montrer $[13,14]$ que la discrimination entre différents modèles de profils de densité dans la zone superficielle ne peut être faite que si la courbe de réflectivité est connue dans un large domaine angulaire au-delà de l'angle critique $\theta_{\mathrm{c}}$. Nous décrivons, dans ce travail, un dispositif et une méthode de mesure destinés à l'étude des surfaces solides et liquides jusqu'aux environs de $10 \theta_{c}$ en utilisant une source de rayons $X$ conventionnelle. 


\section{Dispositif expérimental.}

La seule configuration convenable pour un dispositif expérimental destiné à l'étude de la réflectivité sur la surface libre d'un liquide est celle d'un diffractomètre $\theta-\theta$ à axe horizontal puisque la surface d'un liquide est, par nature, horizontale. Dans ce cas, le tube de rayons $\mathrm{X}$ et le détecteur se déplacent autour d'un axe passant par la surface de l'échantillon maintenu fixe (pour éviter d'avoir à déplacer le compteur, on pourrait utiliser un détecteur linéaire, mais dans notre réalisation nous avons préféré mettre en ouvre un compteur à scintillation et mesurer pas à pas l'intensité réfléchie). La figure 1 . montre les éléments essentiels qui seront décrits par la suite : le goniomètre proprement dit, la source et le détecteur de rayons $\mathrm{X}$, le monochromateur et enfin le porteéchantillon antivibratoire (pour l'étude des liquides).

2.1 Goniomètre. - Il s'agit d'une version légèrement modifiée du diffractomètre $\theta-\theta$ antérieurement réalisé pour l'étude des liquides [15] : le tube à rayons $\mathrm{X}$ et le détecteur sont situés sur les côtés inférieurs d'un losange articulé dont un sommet fixe $\mathrm{O}$ définit l'axe de rotation du goniomètre. $\mathrm{Si}$ l'échantillon est disposé en $\mathrm{O}$, les deux faisceaux incident et réfléchi font sensiblement le même angle $\theta_{1}$ et $\theta_{2}$ avec l'horizontale; ces angles sont mesurés séparément à l'aide de deux capteurs $\mathrm{C}_{1}$ et $\mathrm{C}_{2}$, situés respectivement à l'extrémité du bras porte-tube et sous la fente d'analyse $F_{A}$, placée devant le compteur (Fig. 1). Ces capteurs, de type à transformateur d'induction de résolution infinie, délivrent une tension de sortie proportionnelle au déplacement rectiligne d'un curseur; compte tenu de la position des capteurs et de leur sensibilité, l'erreur commise sur la détermination des angles $\theta_{1}$ et $\theta_{2}$ est inférieure à $3 \times 10^{-2} \mathrm{mrad}$. Il est possible de déplacer l'ensemble fente $F_{A}$-détecteur perpendiculairement au faisceau réfléchi si bien que l'on peut réaliser l'égalité des angles $\theta_{1}$ et $\theta_{2}$ à mieux que $5 \times 10^{-2} \mathrm{mrad}$; ce

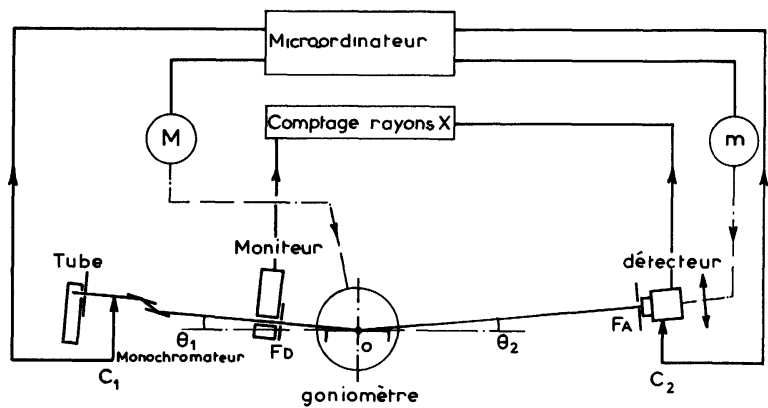

Fig. 1. - Schéma d'ensemble du dispositif de mesure (le moteur $\mathbf{M}$ fait varier simultanément $\theta_{1}$ et $\theta_{2}$; le moteur auxiliaire $\mathrm{m}$ déplace l'ensemble fente d'analyse $\mathrm{F}_{\mathrm{A}}$-détecteur).

[Schematic diagram of the small angle X-ray goniometer (the screw mechanism, driven by the motor $\mathrm{M}$, acts on $\theta_{1}$ and $\theta_{2}$; the motor $\mathrm{m}$ moves $\theta_{2}$ via the $\mathrm{F}_{\mathrm{A}}$-counter system).] mouvement permet en outre l'analyse de l'intensité reçue par le détecteur au voisinage de la réflexion spéculaire, mesure rendue nécessaire pour étudier la réflexion diffuse ou le bruit de fond comme on le verra plus loin.

L'ensemble expérimental est commandé par un microordinateur qui assure aussi le stockage des données.

2. 2 SOURCE ET DÉTECTION DES RAYONS $X$. - Les formules reliant les angles de réflexion à la longueur d'onde [7] montrent que l'on a intérêt à utiliser des rayonnements de grande longueur d'onde : par exemple, l'angle critique $\theta_{\mathrm{c}}$ de réflexion sur la surface libre du mercure est égal à $12 \mathrm{mrad}$ pour le rayonnement $\operatorname{CrK}_{\alpha}(\lambda=0,23 \mathrm{~nm})$ et ne vaut plus que $3,7 \mathrm{mrad}$ pour $\operatorname{MoK}_{\alpha}(\lambda=0,07 \mathrm{~nm})$. Mais nous verrons que l'intensité réfléchie aux grands angles est de plusieurs ordres de grandeur inférieure à l'intensité incidente; or on obtient un meilleur rapport signal/bruit dans la chaîne de détection en utilisant de courtes longueurs d'onde. Dans la pratique, nous avons donc opéré avec le rayonnement $\mathrm{CrK}_{\alpha}$ pour les angles inférieurs à $2 \theta_{\mathrm{c}}$ et avec le rayonnement $\mathrm{CuK}_{\alpha}$ ou $\mathrm{MoK}_{\alpha}$ pour les angles supérieurs. Dans tous les cas, des tubes à foyers fins (dimension du foyer : $8 \times 0,04 \mathrm{~mm}^{2}$ ) ont été utilisés.

Malgré la stabilisation électronique de l'alimentation du tube à rayons $\mathrm{X}$ et la régulation du débit et de la température du circuit de refroidissement, un moniteur (Fig. 1) a été installé pour tenir compte des fluctuations d'intensité à long terme du faisceau incident : la chambre d'ionisation, suivie d'un électromètre et d'un convertisseur tension-fréquence, transmet à la baie de comptage un nombre d'impulsions proportionnel à l'intensité du faisceau incident. Dans ces conditions la reproductibilité des mesures a été trouvée meilleure que $0,5 \%$.

$\mathrm{La}$ détection du rayonnement réfléchi est assurée par un compteur à scintillation NaI. Le signal délivré par le photomultiplicateur est appliqué au discriminateur de hauteur d'impulsions avant d'atteindre l'unité de comptage. Le temps de comptage dépend du nombre présélectionné de photons devant traverser le moniteur et choisi de telle sorte que l'erreur statistique totale soit inférieure à $0,5 \%$; selon les cas, nous avons effectué les expériences en présélectionnant le nombre de photons incidents ou bien le nombre de photons réfléchis.

2.3 Monochromateur. - Pour permettre un changement de longueur d'onde sans modifier la géométrie du goniomètre, le monochromateur est constitué de deux cristaux plans identiques, A et B (Fig. 2), montés dans une chambre maintenue sous vide et munie de deux fenêtres en mylar pour le passage des rayons $\mathrm{X}$. Cette chambre, placée sur le trajet du faisceau incident, peut tourner autour d'un axe $\mathrm{A}_{1} \mathrm{~A}_{1}^{\prime}$ horizontal et perpendiculaire à la direction de propagation $\mathrm{du}$ 


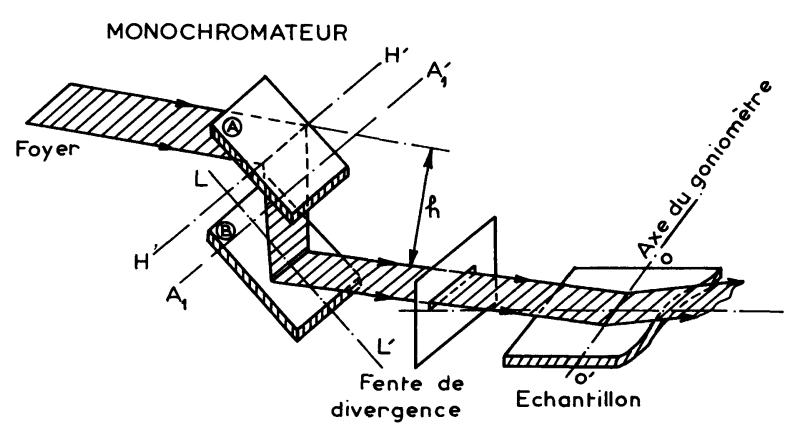

Fig. 2. - Monochromateur à deux cristaux.

[Geometry of the crystal monochromator.]

faisceau pour accorder l'angle de Bragg $\theta_{\mathbf{B}}$ sur la longueur d'onde $\lambda \mathrm{K}_{\alpha}$ caractéristique. Il est possible de déplacer le cristal A par rapport au cristal B selon deux mouvements :

i) une rotation autour d'un axe horizontal $\mathbf{H H}^{\prime}$, également perpendiculaire à la direction de propagation du faisceau (Fig. 2), permet le réglage du parallélisme des plans réticulaires des deux cristaux,

ii) une translation le long d'une direction perpendiculaire au cristal B permet d'ajuster le décalage $h$ entre le faisceau incident et le faisceau émergent, $h$ étant relié à la distance $D$ séparant les deux cristaux par la relation $h=2 D \cos \theta_{\mathbf{B}}$.

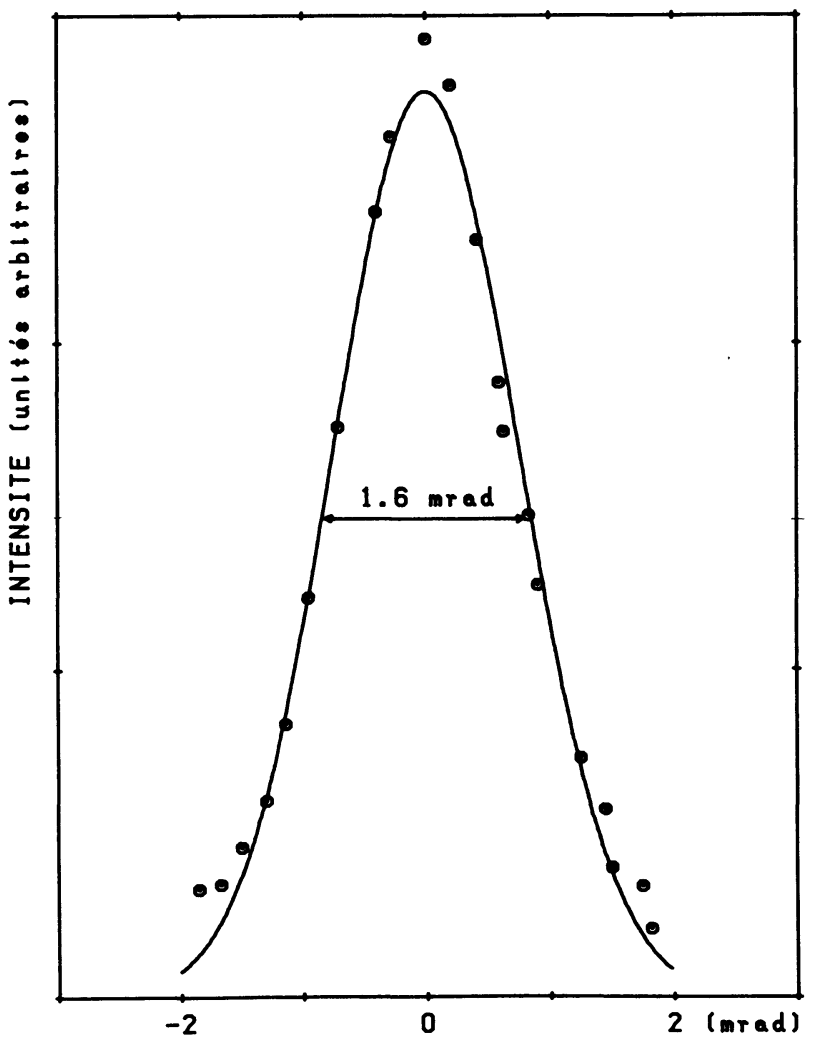

Fig. 3. - Profil de réflexion intrinsèque du monochromateur.

[Rocking curve of the monochromator.]
En outre, le cristal B peut osciller autour de son axe longitudinal $L^{\prime} L^{\prime}$ : il est ainsi possible d'ajuster la direction du faisceau diffracté et de rendre le plan de réflexion perpendiculaire à l'axe $0^{\prime}$ ' du goniomètre.

Les monocristaux A et B sont en fluorure de lithium, matériau choisi en fonction de sa forte brillance pour une mosaïcité donnée. On sait que la bande passante (rocking curve) des cristaux dépend de la qualité de ceux-ci et de leur état de surface : après installation et alignement, la rocking curve du double-monochromateur (Fig. 3) a une largeur a mi-hauteur de 1,6 mrad. La raie $K_{\alpha_{1}}$ du rayonnement (Fig. 4, courbe en trait continu) est isolée à l'aide d'une fente de divergence $F_{D}$ située devant l'échantillon (Figs. 1 et 2).

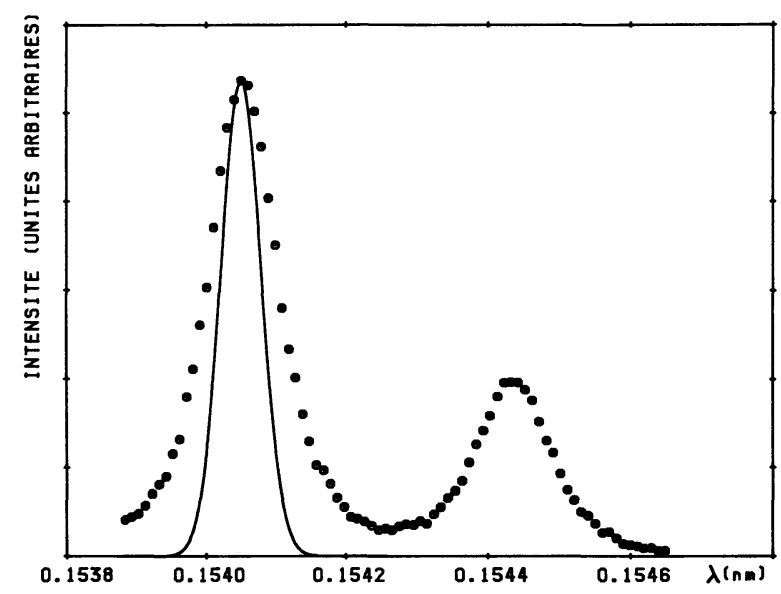

Fig. 4. - Caractéristique du faisceau incident : doublet $K_{\alpha_{1}}-K_{\alpha_{2}}$ (cercles) et radiation isolée par la fente de divergence $F_{D}$ (trait continu).

[A plot of the intensity distribution in the incident beam : $\mathrm{K}_{\alpha_{1} \alpha_{2}}$ wavelengths (circles) and $\mathrm{K}_{\alpha_{1}}$ radiation isolated by the divergence $F_{D}$ slit (solid line).]

2.4 Porte-ÉChantillon antiviBratoire. - L'étude des liquides nécessite l'emploi d'un dispositif permettant d'éliminer les vibrations de la surface libre. La méthode que nous avons adoptée consiste à désolidariser l'échantillon du goniomètre grâce à un dispositif dont le rôle est d'amortir les vibrations de l'échantillon et de maintenir la surface réfléchissante dans l'axe de rotation du goniomètre.

Le dispositif d'amortissement (Fig. 5) est du type filtre passe-bas : le porte-échantillon et l'échantillon liquide sont suspendus par l'intermédiaire d'un ressort $R$ en un point fixe $O_{1}$ solidaire de la table supportant le goniomètre. L'amortissement des oscillations de l'ensemble mobile est réalisé par les courants induits dans une bobine $B_{0}$, placée dans une induction magnétique, dont le circuit extérieur est fermé sur une résistance ajustable $r$. A cet effet, le porte-échantillon comporte à sa partie inférieure un cylindre sur lequel plusieurs spires de fil de cuivre sont enroulées; 


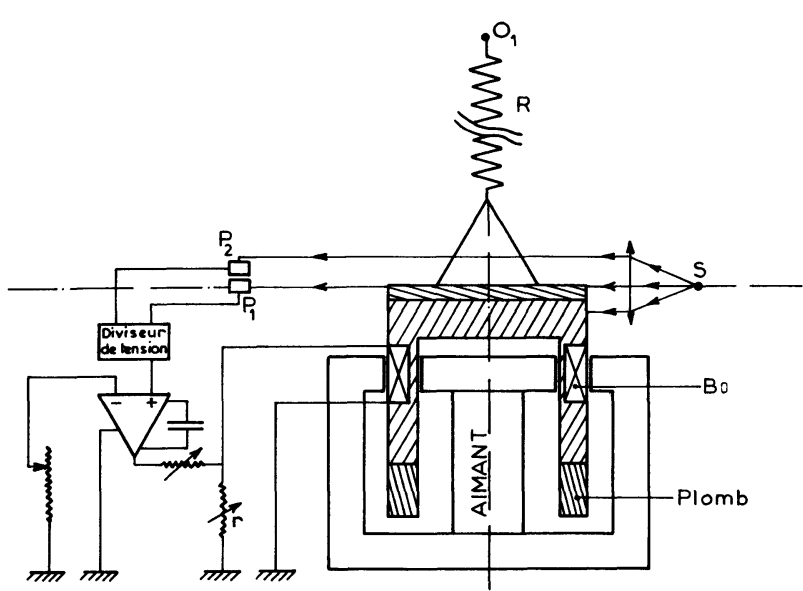

Fig. 5. - Schéma du porte-échantillon antivibratoire et du dispositif d'asservissement en hauteur.

[Scheme of the sample suspension system and the optical servo-mechanism for the surface level position.]

la bobine est située dans l'entrefer d'un aimant permanent. Selon la raideur du ressort, il est nécessaire de lester l'ensemble mobile en ajoutant un cylindre de plomb pour obtenir la fréquence de coupure minimale, typiquement de l'ordre de $1 \mathrm{~Hz}$; l'amortissement est réglé à la valeur correspondant au régime critique en ajustant la résistance extérieure $r$ (Fig. 5).

Ce dispositif a une autre fonction : celle de compenser l'allongement éventuel du ressort ou bien l'évaporation lente d'un échantillon liquide grâce à un asservissement en hauteur qui maintient la position de la surface libre de l'échantillon constante par rapport à l'axe du goniomètre. Un faisceau lumineux, issu de la source $S$ (Fig. 5) et se propageant perpendiculairement à la direction des rayons $\mathrm{X}$, éclaire deux cellules photoélectriques $P_{1}$ et $P_{2}$. Par construction, $P_{1}$ est située dans l'axe du goniomètre et, à l'équilibre, le courant $I$ parcourant la bobine $\mathrm{B}_{0}$ est ajusté de telle sorte que la surface libre intercepte partiellement le faisceau lumineux; $P_{1}$ est alors à demi-éclairée. La cellule $P_{2}$, constamment éclairée, permet de s'affranchir des fluctuations d'intensité de la source lumineuse. Un asservissement à action proportionnelle et intégrale, commandé par la tension de sortie d'un circuit électronique diviseur de tension auquel sont reliées les cellules $P_{1}$ et $P_{2}$, ajuste le courant $I$ : la surface libre de l'échantillon peut ainsi être maintenue à mieux de $3 \mu \mathrm{m}$ dans l'axe $\mathrm{du}$ goniomètre pendant une période de plusieurs jours.

\section{Exemples d'application.}

Pour les rayonnements électromagnétiques de courtes longueurs d'onde, l'indice de réfraction $n=1-\delta-i \beta$ des matériaux condensés est légèrement inférieur à 1 et si le rayonnement provient d'un milieu d'indice égal à 1 (vide ou vapeur) il sera presque totalement réfléchi tant que l'angle d'attaque restera inférieur à l'angle critique $\theta_{\mathrm{c}}=\sqrt{2 \delta}$ (la réflexion est totale lorsque $\beta=0$ ). Le principe de la méthode [8] consiste à mesurer en fonction de l'angle d'attaque l'intensité réfléchie et à en déduire la variation de l'indice de réfraction dans la zone de transition séparant les deux milieux. Il est alors facile de calculer le profil de densité.

Sauf dans le cas où l'on se limite à la détermination des deux paramètres $\beta$ et $\delta$ servant à calculer l'indice de réfraction massique, il est généralement nécessaire de déterminer l'intensité réfléchie jusqu'à des angles beaucoup plus grands que l'angle critique $\theta_{\mathrm{c}}$. Par exemple, les mesures doivent être effectuées dans un domaine angulaire supérieur à $5 \theta_{\mathrm{c}}$ si l'on veut distinguer un profil de densité oscillatoire d'un profil de type tangente hyperbolique [13].

Cependant, au-delà de $\theta_{\mathrm{c}}$, l'intensité réfléchie varie sensiblement comme $\theta^{-4}$ et il devient impératif de déterminer avec précision la diffusion parasite et le bruit de fond.

i) On peut installer devant le détecteur une fente d'analyse $F_{A}$ de divergence supérieure à la divergence du faisceau réfléchi et mesurer successivement l'intensité réfléchie spéculairement à un angle $\theta_{2}$ donné $\left(\theta_{2}=\theta_{1}\right)$ puis l'intensité relative au bruit de fond à un angle voisin de $\theta_{2}$ situé en dehors de la réflexion spéculaire.

C'est la méthode que nous avons utilisée pour déterminer, par exemple, la réflectance de l'eau jusqu'à $2 \theta_{c}$ (Fig. 6). La valeur $\delta=7,78 \times 10^{-6}$ déterminée expérimentalement en utilisant le rayonnement $\mathrm{K}_{\alpha}$ du chrome est en bon accord avec la valeur calculée $\delta=7,94 \times 10^{-6}$. La réflectance ne dépend sensiblement de $\beta$ que pour des angles d'attaque inférieurs à $\theta_{c}$; en raison des dimensions limitées de l'échantillon qui entraînent une déformation de la courbe de réflectivité aux faibles angles (Fig. 6), $\beta$ ne peut être déterminé, dans cet exemple, qu'avec une précision médiocre.

ii) Généralement, nous avons préféré utiliser une fente d'analyse fine (divergence $0,05 \mathrm{mrad}$ ) et mesurer, autour de chaque réflexion spéculaire, l'intensité du faisceau réfléchi depuis l'angle $\left(\theta_{2}-\Delta \theta\right)$ jusqu'à l'angle $\left(\theta_{2}+\Delta \theta\right), \Delta \theta$ étant choisi suffisamment grand pour que l'intensité mesurée ne dépende plus de l'angle. La figure 7 montre l'allure des courbes obtenues, par exemple à trois angles d'attaque différents, et la totalité de la courbe de réflectivité du glycérol déterminée à l'aide dụ rayonnement $K_{\alpha}$ du cuivre selon cette méthode; (l'intensité réfléchie prise en considération est proportionnelle à l'aire sous la courbe $\left.I=f\left(\theta_{2}\right)\right)$. Le profil de densité dans la zone interfaciale séparant le liquide de l'air semble être de la forme tangente hyperbolique comme l'indique, au moins dans le domaine angulaire exploré, l'accord entre la courbe de réflectivité mesurée et celle calculée [9] à partir d'un profil de largeur $L=1,56 \mathrm{~nm}$ (Fig. 7). Il est à remarquer que cette valeur est nettement supérieure à la valeur $L=0,37 \mathrm{~nm}$ calculée à $288 \mathrm{~K}$ à partir 


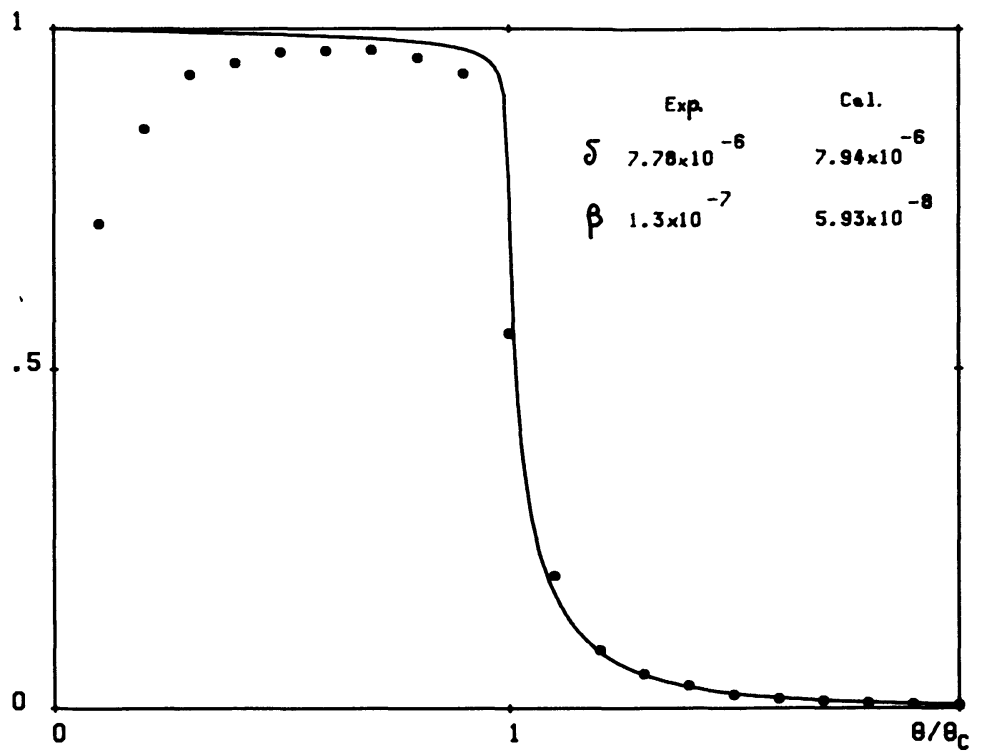

Fig. 6. - Courbe de réflectivité de l'eau mesurée à la température ambiante à l'aide du rayonnement $\mathrm{CrK}_{\alpha}$. En trait plein : courbe calculée.

[X-ray reflectivity curve of water at $293 \mathrm{~K}$ using $\mathrm{CrK}_{\alpha}$ radiation. Solid line : the reflectivity curve predicted by the sharp boundary model.]

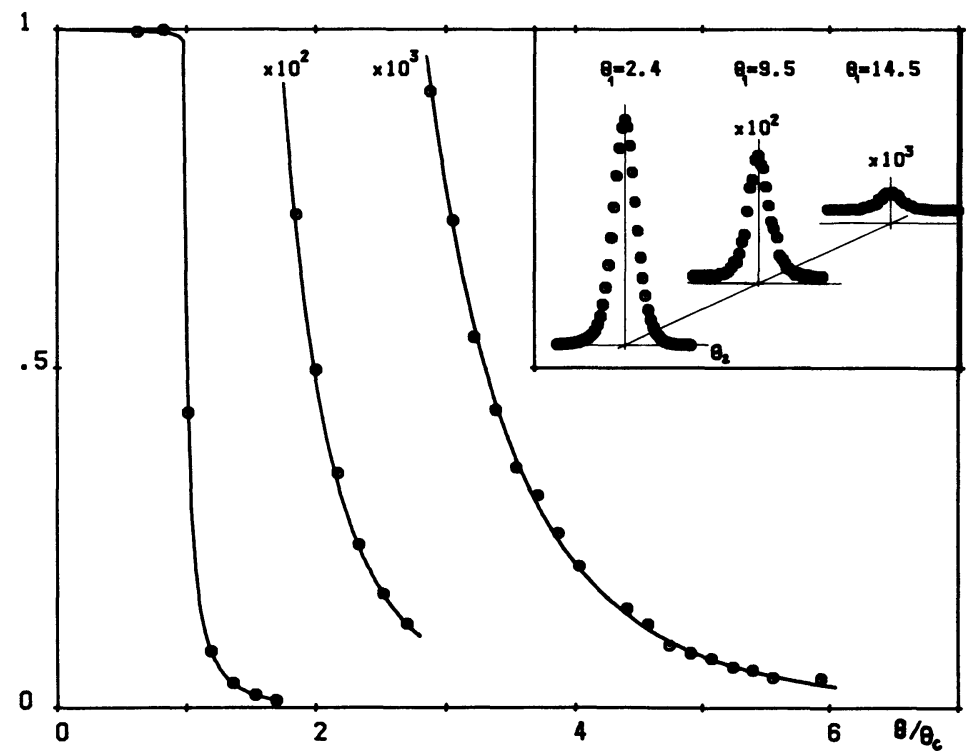

Fig. 7. - Courbe de réflectivité du glycérol, mesurée à la température ambiante à l'aide du rayonnement $\mathrm{CuK}_{\alpha_{1}}(\mathrm{cercles})$. En trait plein : courbe de réflectivité calculée en admettant un profil de densité de type tangente hyperbolique (En encart : intensité réfléchie au voisinage de 3 réflexions spéculaires).

[X-ray reflectivity curve of glycerol at $293 \mathrm{~K}$ using $\mathrm{CuK}_{\alpha_{1}}$ radiation as-measured (circles) and calculated assuming a hyperbolic tangent profile (solid line). In inset : scans obtained at three angles (in mrad) as a function of the deviation from the specular angle.]

de la relation de Mon et Stroud $L=24 K_{\mathrm{T}} \sigma$ [16] dans l'hypothèse où cette théorie reliant la largeur du profil de densité à la compressibilité $K_{\mathrm{T}}$ et à la tension superficielle $\sigma$, en prenant $K_{\mathrm{T}}=2,2 \times 10^{-11} \mathrm{~cm}^{2} \cdot \mathrm{dyn}^{-1}$ et $\sigma=64$ dyn. $\mathrm{cm}^{-1}$ [17], est applicable au cas du glycérol.

Rappelons que nous avons déterminé la courbe de réflectivité du mercure liquide jusqu'à $10 \theta_{\mathrm{c}}$ en utilisant le rayonnement $K_{\alpha}$ du molybdène et que nous avons été amenés à conclure à l'existence d'un profil nonmonotone dans la zone de transition liquide-vapeur [14].

La méthode de mesure qui consiste à analyser chacune des réflexions permet d'avoir des informations 
sur la rugosité de la surface et sur la stratification de la couche superficielle [18] : si le faisceau réfléchi par une surface liquide (Fig. 8, courbe b) a sensiblement la même divergence que le faisceau incident (Fig. 8, courbe a) - à condition d'avoir correctement amorti les ondulations de surface - pour un solide, la présence d'imperfections de surface entraîne l'apparition de réflexions diffuses; la courbe c (Fig. 8) montre l'allure d'une réflexion sur la surface d'un échantillon constitué d'un film de nickel (épaisseur : $68 \mathrm{~nm}$ ) déposé sur un support de silice polie optiquement. On remarquera sur la courbe de réflectivité relative à cet échantillon (Fig. 9) l'apparition des franges de Kiessig au-delà de l'angle critique de réflexion totale; on sait que ces interférences permettent une détermination précise de l'épaisseur des dépôts.

\section{Conclusion.}

Nous avons décrit un dispositif permettant la détermination des courbes de réflectivité des rayons $X$ sur des surfaces solides, géométriquement planes, ou liquides de quelques $\mathrm{cm}^{2}$ : les performances de l'appareillage sont telles que les mesures peuvent être effectuées bien au-delà de l'angle critique $\theta_{\mathrm{c}}$, dans un domaine angulaire jusque-là inexploré en raison du faible rapport signal/bruit $\left({ }^{*}\right)$.

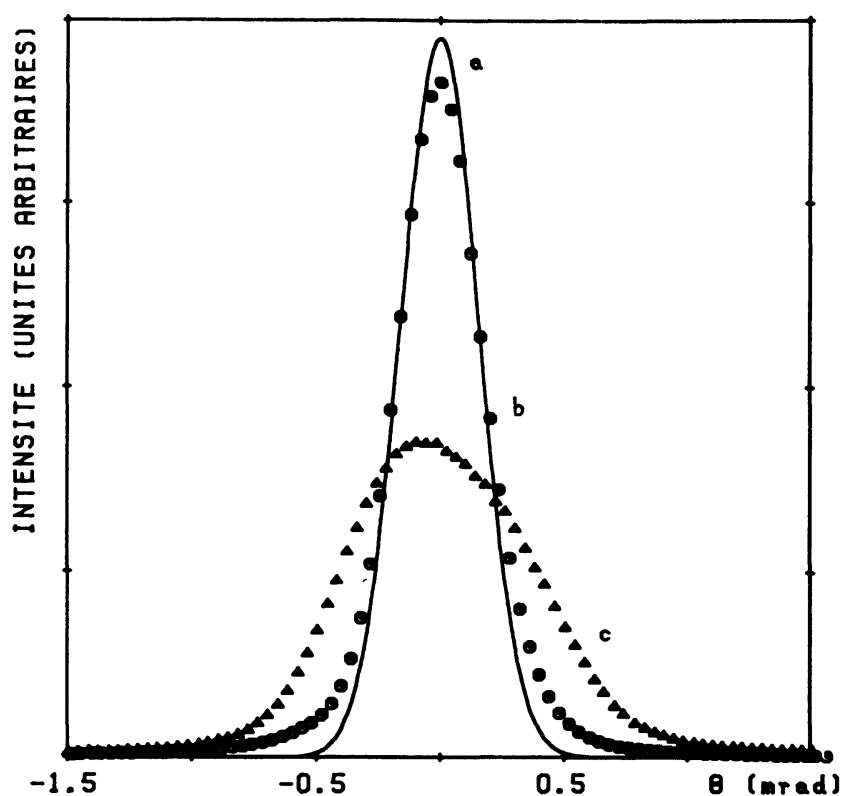

Fig. 8. - Comparaison entre la divergence du faisceau incident (courbe a, trait continu) et celle du faisceau réfléchi par un liquide (courbe $b$, cercles) et un solide poli optiquement (courbe $c$, triangles).

[Comparison between the divergence of the incident beam (solid line) and the divergence of the reflected beam : from a liquid (circles) or from a solid surface (triangles).]

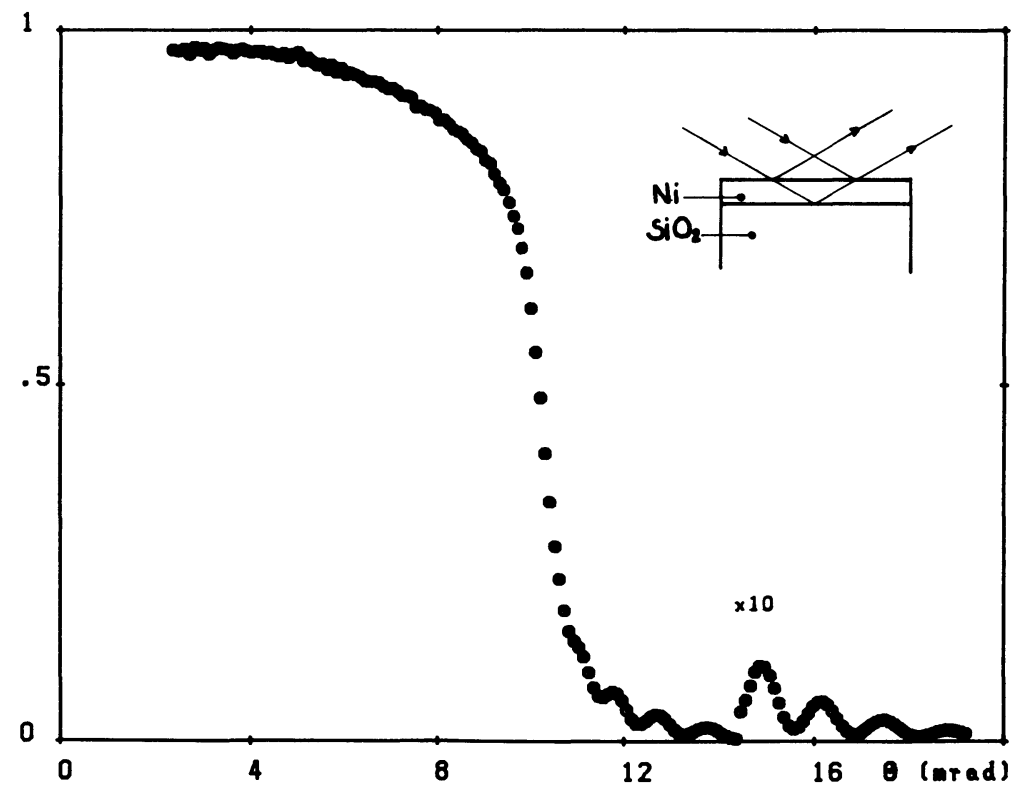

Fig. 9. - Courbe de réflectivité d'un film de nickel (épaisseur $68 \mathrm{~nm}$ ) déposé sur un substrat de silice fondue, mesurée à l'aide du rayonnement $\mathrm{CrK}_{\alpha_{1}}$.

[Intensity reflected from a $68 \mathrm{~nm}$-thick $\mathrm{Ni}$ film deposited on a silica glass $\left(\mathrm{CrK}_{\alpha_{1}}\right.$ radiation).]

$\left(^{*}\right)$ Note ajoutée aux épreuves : l'intervalle angulaire dans lequel les mesures de réflectivité sont effectuées peut être considérablement augmenté par l'emploi d'une source de rayons $\mathrm{X}$ à haut flux : c'est ce qui a été montré par BrASlau, A., Deutsch, M., Pershan, P. S. et Weiss, A. H., Phys. Rev. Lett. 54 (1985) 114.
L'interprétation des données ainsi obtenues permet d'avoir des informations sur le profil de densité dañs la zone interfaciale séparant le matériau condensé du milieu extérieur et, par là, des informations indirectes sur la structure de l'interface. 
Mais l'application de la réflexion des rayons $\mathrm{X}$ peut ne pas se limiter à la détermination des réflectances en mesurant l'intensité réfléchie en fonction de l'angle d'attaque : on peut aussi maintenir l'angle d'incidence fixe (voisin de l'angle critique $\theta_{\mathrm{c}}$ ) et

- mesurer l'intensité diffractée superficiellement; jusqu'à présent les seuls résultats dont on dispose concerne des surfaces monocristallines [3] mais il semble possible d'envisager une extension de cette méthode à des poudres ou à des liquides [19].

- ou bien opérer à énergie incidente variable (au voisinage d'un seuil d'absorption) et obtenir des spectres de structure fine d'absorption X [2,20]. Dans une prochaine publication nous décrirons le dispositif de RéflEXAFS adapté à ce genre d'étude.

\section{Bibliographie}

[1] Block, A. N. et Rice, S. A., Phys. Rev. 185 (1969) 933. Inagaki, T., Arakawa, E. T. et Williams, M. W., Phys. Rev. B 23 (1981) 5246.

Greuter, F., Gubler, U., Krieg, J., Preiswerk, H. et Oelhafen, P., Surf. Sci. 124 (1983) 489.

[2] Martens, G. et Rabe, P., J. Phys. C : Solid State Phys. 14 (1981) 1523.

[3] Afanasiev, A. M. et Melkonyan, M. K., Acta Cryst. A 39 (1983) 207.

[4] Cowan, P. L., Golovchenko, J. A. et Robbins, M. F., Phys. Rev. Lett. 44 (1980) 1680.

[5] Klein, A. G. et Werner, S. A., Rep. Prog. Phys. 46 (1983) 259.

[6] Compton, A. H. et Allison, S. K., X-rays in Theory an Experiment (D. Van. Nostrand. Company, Inc., N.Y.) 1935.

[7] Parratt, L. G., Phys. Rev. 95 (1954) 359.

[8] Névot, L. et Croce, P., Rev. Phys. Appl. 15 (1980) 761.

[9] Smirnov, L. A. et Anokhin, S. B., Opt. Spectrosc. (USSR) 48 (1980) 315.
[10] Steps, H., Ann. Phys. Fr. 16 (1933) 949.

[11] Lu, B. C. et Rice, S. A., J. Chem. Phys. 68 (1978) 5558.

[12] Sluis, D., D'Evelyn, M. P. et Rice, S. A., J. Chem. Phys. 78 (1983) 1611.

[13] D'Evelyn, M. P. et Rice, S. A., J. Chem. Phys. 78 (1983) 5081.

[14] Bosio, L. et Oumezine, M., J. Chem. Phys. 80 (1984) 959.

[15] Bizid, A., Bosio, L., Cortès, R. et Defrain, A., Bull. Soc. Fr. Minéral. Cristal. 100 (1977) 9.

[16] Mon, K. K. et Stroud, D., Phys. Rev. Lett. 45 (1980) 817.

[17] Sanchez, I. C., J. Chem. Phys. 79 (1983) 405.

[18] Guentert, O. J., Phys. Rev. 138 (1965) A 732.

De Korte, P. A. J. et Lainé, R., Appl. Optics 18 (1979) 236.

[19] Mohanty, U. et Rice, S. A., J. Chem. Phys. 79 (1983) 2482.

[20] Bosio, L., Cortès, R., Defrain, A. et Froment, M., J. Electroanal. Chem. 180 (1984) 265. 